\title{
OVERLAP PROBLEMS ON THE CIRCLE
}

\author{
S. JUNEJA, ${ }^{*}$ Tata Institute for Fundamental Research \\ M. MANDJES, ${ }^{* *}$ University of Amsterdam, EURANDOM and \\ Eindhoven University of Technology
}

\begin{abstract}
Consider a circle with perimeter $N>1$ on which $k<N$ segments of length 1 are sampled in an independent and identically distributed manner. In this paper we study the probability $\pi(k, N)$ that these $k$ segments do not overlap; the density $\varphi(\cdot)$ of the position of the disks on the circle is arbitrary (that is, it is not necessarily assumed uniform). Two scaling regimes are considered. In the first we set $k \equiv a \sqrt{N}$, and it turns out that the probability of interest converges $(N \rightarrow \infty)$ to an explicitly given positive constant that reflects the impact of the density $\varphi(\cdot)$. In the other regime $k$ scales as $a N$, and the nonoverlap probability decays essentially exponentially; we give the associated decay rate as the solution to a variational problem. Several additional ramifications are presented.
\end{abstract}

Keywords: Overlap probability; asymptotics; nonuniformity

2010 Mathematics Subject Classification: Primary 60C05; 60F05; 60F10; 65C05

\section{Introduction}

In this paper we study overlap problems of the following type. Consider an interval of length $N>1$. Then $k \leq N$ points are sampled independently in this interval, in the simplest model uniformly at random. These $k$ points are the midpoints of $k$ intervals of length 1 (to be referred to throughout this paper as 'disks'). The interval should be thought of as 'circular', in the sense that, for instance, a disk with midpoint $\frac{1}{4}$ covers $\left[0, \frac{3}{4}\right]$ as well as $\left[N-\frac{1}{4}, N\right]$.

The primary objective of this paper is to analyze the probability $\pi(k, N)$ that the $k$ disks do not overlap (the 'nonoverlap probability') — several applications could be thought of, such as in wireless communications. This problem has been studied in detail in the 'uniform case' described above, that is, the case in which all midpoints are independent and identically distributed (i.i.d.) samples from the homogeneous distribution on $[0, N]$; see, e.g. [2, p. 137]. The analysis complicates substantially in the case when the midpoints are i.i.d. samples from an arbitrary distribution on $[0, N]$, say $\varphi_{N}(\cdot)$.

We consider two asymptotic regimes: in the first $k$ scales as $a \sqrt{N}$ ('square-root scaling') whereas in the second $k$ scales as $a N$ ('linear scaling'), for some scalar $a>0$. In both cases we let $N \rightarrow \infty$, where it is assumed that $\varphi_{N}(x)=N^{-1} \varphi(x N)$, where $\varphi(\cdot)$ is a density on $[0,1]$. Under the square-root scaling, we obtain

$$
\pi(a \sqrt{N}, N) \rightarrow \exp \left(-a^{2} \int_{0}^{1}(\varphi(x))^{2} \mathrm{~d} x\right) \text { as } N \rightarrow \infty,
$$

Received 12 July 2011; revision received 11 April 2012.

* Postal address: Tata Institute for Fundamental Research, Homi Bhabha Road, Colaba Mumbai, India.

Email address: juneja@tifr.res.in

** Postal address: Korteweg-de Vries Institute for Mathematics, University of Amsterdam, Science Park 904, Amsterdam 1098 XH, The Netherlands. Email address: m.r.h.mandjes@uva.nl 
under mild regularity conditions. It is an easy exercise to show that the expression on the lefthand side is maximized by $\varphi(x)=1$, i.e. when the midpoints of the disks are thrown uniformly on the circle, the nonoverlap probability is maximized. Informally, the more 'clustered' the density, the lower the nonoverlap probability. The proof of the above statement relies on first establishing the claim for piecewise-constant densities, and then performing a limiting argument.

In the linear regime, we prove that the nonoverlap probability decays essentially exponentially, where the decay rate is characterized as the solution to a variational problem. In addition, we compute the number $F_{j}(N) / N$ as $N \rightarrow \infty$, where $F_{j}(N) / N$ is the fraction of the perimeter that is covered by $j$ disks. It is noted that our results cover all scalings between square-root scaling and linear scaling as well: in addition to the findings mentioned above, we also identify the asymptotics of $\pi\left(a N^{\alpha}, N\right)$ for $\alpha \in\left(\frac{1}{2}, 1\right)$.

There is a striking similarity between the problems sketched above and their 'discrete counterparts': then the $k$ samples ('birthdays') are (uniformly or nonuniformly) distributed on $\{1, \ldots, N\}$. Much attention has been paid to computing the probability $\pi_{b}(k, N)$ that all $k$ samples are distinct; this setting is known as the 'birthday problem', and goes back to at least Von Mises [19]. There is a well-known expression for the 'uniqueness probability' (or 'no-coincidence probability') in the case when all outcomes are equally likely:

$$
\pi_{b}(k, N)=\frac{N ! /(N-k) !}{N^{k}} .
$$

For the square-root scaling, it is a matter of elementary calculus to prove that there is a finite, positive limit: $\pi(a \sqrt{N}, N) \rightarrow \exp \left(-a^{2} / 2\right)$; under the linear scaling, there is essentially exponential decay with an explicitly given decay rate.

Considerably less is known for the case in which the outcomes are not equally probable. In general, computing the uniqueness probability in this nonuniform setting is computationally demanding — see, however, for an efficient evaluation scheme, [11], and in addition interesting results can be found in [8] and [14]. For the case of a piecewise-uniform density, relatively explicit results can be derived though. In such a piecewise-uniform scenario there are $F_{i}$ (out of $N$ ) outcomes that have probability $\alpha_{i} / N$, with obviously $F_{1}+\cdots+F_{d}=N$ and $\alpha_{1} F_{1}+\cdots+\alpha_{d} F_{d}=N, d \in \mathbb{N}$. Both for the square-root scaling [5] and linear scaling [13], asymptotics have been derived that can be viewed as generalizations of the asymptotics for the uniform case, as discussed above. It turns out that many results that have been derived for the discrete birthday problem have a natural analogue in its continuous counterpart; in the present paper we establish these analogues.

There is a rich and substantial literature in statistical physics related to the problem that we consider. This branch goes back to at least Tonks [18], who considered the problem of $N$ disks (atoms in his terminology) of a given radius uniformly distributed along a single dimensional line segment. In this setup, expressions are derived for a number of statistical and thermodynamical quantities. In particular, the probability that the $N$ disks do not intersect is easily computed from his analysis. Tonks' work has been generalized in many ways. Some of the early important contributions include [9], [16], and [17]—see [12, Chapter 1] for an overview. Essentially, these considered particles on the real line with interaction between nearest neighbors. For a specified potential function between interacting neighbors, the overall partition function is analyzed. In many cases, the Laplace transform of this function can be computed. Through appropriate inversion of this transform, various statistical and thermodynamic quantities of the system can be identified; in particular, Tonks' model becomes a special case of such an analysis. While it is conceivable that such an analysis can be used to characterize the probability that $N$ disks of 
random lengths thrown on a one-dimensional circle using a general distribution do not intersect, to the best of our knowledge this has not been done in the literature. At the methodological level our paper also contributes to the existing literature: we develop an elementary technique that extends results for the uniform case to the nonuniform case, which we feel has the potential of being more broadly applicable.

A substantial, relatively recent branch of the statistical physics literature considers a related problem known as random sequential adsorption (RSA). There particles are thrown sequentially uniformly on a line segment (also on lattices and in more general spaces), the overlapping particles are ignored, and the system time to jamming as well as related performance measures are analyzed. Jamming occurs when the line segment has no space available to accommodate further particles. In the continuous setting, this is also known as the car parking problem (as was introduced by Rényi [15]); Evans [7] and Bartelt and Privman [3] provided excellent surveys of this strand of literature. See [4] and the references therein for some recent work that allows particles to be distributed nonuniformly. However, this line of work primarily focuses on other metrics than ours, in that it is not directly applicable to the overlap probability that we consider.

This paper is organized as follows. In Section 2 we point out that the problem sketched above can be interpreted as a variant of the classical birthday problem, in terms of its continuous counterpart. This section also contains a number of preliminary results that are used several times later in the paper. Section 3 deals with the case in which the density $\varphi(\cdot)$ is piecewise constant; essentially the techniques from [13] can be reused here. Then in Section 4 these results are used to address the case of an arbitrary density $\varphi(\cdot)$. In Sections 3 and 4 both the square-root scaling and linear scaling are covered. Concluding remarks are given in Section 5.

\section{Representation as 'continuous' birthday problem; limiting regimes}

In this section we first give an insightful derivation of the known result that in the uniform case (that is, $\varphi(x)=1$ for $x \in[0,1])$ the nonoverlap probability $\pi(k, N)$ equals $(1-k / N)^{k-1}$, relying on the connection with discrete birthday problems. Then we study various asymptotic regimes, and present a number of additional preliminaries.

\subsection{Expression for the nonoverlap probability}

Abramson and Moser [1] proved the following result. Let there be $N$ different birthdays, $k$ people, and consider the probability $\varrho(k, N, \ell)$ that all birthdays have to be at least $\ell$ days apart. A combinatorial argument yields

$$
\varrho(k, N, \ell)=\frac{(N-k(\ell-1)-1) !}{N^{k-1}(N-k \ell) !} ;
$$

it is easily shown that upon choosing $\ell=1$ we retrieve the solution to the 'classical' birthday problem.

Interestingly, we can compute the nonoverlap probability in our continuous model as a limit:

$$
\pi(k, N)=\lim _{m \rightarrow \infty} \varrho(k, N m, m)
$$

'Stirling' gives

$$
\begin{aligned}
\lim _{m \rightarrow \infty} & \frac{(N m-k(m-1)-1) !}{(N m)^{k-1}(N m-k m) !} \\
= & \mathrm{e}^{-k+1} \lim _{m \rightarrow \infty}\left(1-\frac{k(m-1)+1}{N m}\right)^{k-1}\left(1+\frac{k-1}{(N-k) m}\right)^{(N-k) m}
\end{aligned}
$$


After standard algebra we obtain

$$
\pi(k, N)=\left(1-\frac{k}{N}\right)^{k-1} .
$$

It is noted that this is a standard result; see, e.g. [2, p. 137]. We decided to include the above derivation as it well reflects the relation with the discrete birthday problem.

Remark 1. It is insightful to contrast the above formula with the one for the discrete birthday problem, say, $\pi_{b}(k, N)$. In [6] it is argued that

$$
\begin{aligned}
\pi_{b}(k, N) & =\frac{N ! /(N-k) !}{N^{k}} \\
& =\prod_{i=0}^{k-1}\left(1-\frac{i}{N}\right) \\
& =\exp \left(\sum_{i=0}^{k-1} \log \left(1-\frac{i}{N}\right)\right) \\
& \approx \exp \left(-\sum_{i=0}^{k-1} \frac{i}{N}\right) \\
& \approx \mathrm{e}^{-k^{2} /(2 N)} .
\end{aligned}
$$

For our continuous variant, we observe that

$$
\pi(k, N)=\exp \left((k-1) \log \left(1-\frac{k}{N}\right)\right) \approx \mathrm{e}^{-k^{2} / N} .
$$

In other words, $\pi(k, N)$ is roughly the square of $\pi(k, N)$. We will encounter this phenomenon more often in this paper. The following intuitive reason can be given. In the discrete birthday problem any person 'blocks' one day for all other people: the second person entering has a probability of $1-1 / N$ of no overlap with the first person. In the continuous counterpart, on the contrary, any person essentially blocks an interval of length 2: the second person entering has a probability of $1-2 / N$ of no overlap with the first person.

Remark 2. As noted in the introduction, we considered the 'cyclic' nonoverlap probability. The corresponding noncyclic probability can be directly derived from this. Define $\pi^{\prime}(k, N)$ as the noncyclic nonoverlap probability when throwing $k$ midpoints of disks uniformly on the interval $[0, N]$, where the endpoints 0 and $N$ are not allowed to be covered by a disk. It is seen immediately that

$$
\pi(k, N)=\left(\frac{N-1}{N}\right)^{k-1} \pi^{\prime}(k-1, N-1) .
$$

In addition, define $\pi^{\prime \prime}(k, N)$ as the noncyclic nonoverlap probability when throwing $k$ midpoints of disks uniformly on the interval $[0, N]$, where the endpoints 0 and $N$ are allowed to be covered by a disk. Then

$$
\pi^{\prime}(k, N)=\left(\frac{N-1}{N}\right)^{k} \pi^{\prime \prime}(k, N-1) .
$$


It now follows that

$$
\pi^{\prime}(k, N)=\left(1-\frac{k}{N}\right)^{k}, \quad \pi^{\prime \prime}(k, N)=\left(1-\frac{k-1}{N}\right)^{k}
$$

In addition,

$$
\pi^{\prime}(k, N) \leq \pi(k, N) \leq \pi^{\prime \prime}(k, N)
$$

\subsection{Asymptotics under various scalings}

In this subsection we introduce the scalings considered in this paper: the square-root scaling (that is, $k=a \sqrt{N}$ ) and the linear scaling (that is, $k=a N$ with $a<1$ ). For both scalings, we study the behavior of the nonoverlap probability for large $N$.

First we focus on the square-root scaling. Observe that

$$
\pi(a \sqrt{N}, N)=\left(1-\frac{a}{\sqrt{N}}\right)^{a \sqrt{N}-1} \rightarrow \mathrm{e}^{-a^{2}}
$$

as $N \rightarrow \infty$, which is again the square of the outcome in the corresponding discrete counterpart.

Furthermore, for the linear scaling, we have

$$
\pi(a N, N)=(1-a)^{a N-1},
$$

that is, $\pi(a N, N)$ decays exponentially in $N$. This means that

$$
N^{-1} \log \pi(a N, N) \rightarrow a \log (1-a)=: \zeta_{0} \quad \text { as } N \rightarrow \infty .
$$

The decay rate $\zeta_{0}$ looks like $-a^{2}$ for small $a$, in line with the result for $\pi(a \sqrt{N}, N)$. In the discrete birthday problem we found that $N^{-1} \log \pi(a N, N) \approx-a^{2} / 2$ for large $N$ and small $a$, so again the 'squaring rule' applies.

The following general statement can be made. For $\alpha \in[0,1)$,

$$
\pi\left(a N^{\alpha}, N\right) \approx\left[\left(1-\frac{a}{N^{1-\alpha}}\right)^{N^{1-\alpha} / a}\right]^{a^{2} N^{2 \alpha-1}} \approx \mathrm{e}^{-a^{2} N^{2 \alpha-1}},
$$

which leads to the limiting value 1 for $\alpha$ below $\frac{1}{2}$, and 0 for $\alpha$ above $\frac{1}{2}$.

The following lemma is an immediate consequence of inequality (3).

Lemma 1. In the square-root scaling,

$$
\lim _{N \rightarrow \infty} \pi(a \sqrt{N}, N)=\lim _{N \rightarrow \infty} \pi^{\prime}(a \sqrt{N}, N)=\lim _{N \rightarrow \infty} \pi^{\prime \prime}(a \sqrt{N}, N)=\mathrm{e}^{-a^{2}} .
$$

In the linear scaling,

$$
\lim _{N \rightarrow \infty} \frac{1}{N} \log \pi(a N, N)=\lim _{N \rightarrow \infty} \frac{1}{N} \log \pi^{\prime}(a N, N)=\lim _{N \rightarrow \infty} \frac{1}{N} \log \pi^{\prime \prime}(a N, N)=\zeta_{0} .
$$




\subsection{General disk size}

Interestingly, the case of random disk sizes can also be dealt with. To show how this works, we first re-prove (1), and then explain how this argument can be extended to cover random disk sizes as well. We emphasize that the results in later sections of this paper consider the standard case of disks of size 1 .

For this, select a disk at random and mark its center as 0 . Let $X_{(1)}, \ldots, X_{(k-1)}$ denote the clockwise distance from 0 of the centers on the circle of the remaining $k-1$ disks marked in an ascending order. Note that $\left(X_{(1)}, \ldots, X_{(k-1)}\right)$ are distributed as the order statistics of $k-1$ independent, uniformly distributed random variables. Clearly,

$$
\pi(k, N)=\mathbb{P}\left(X_{(1)}>1, X_{(2)}>X_{(1)}+1, \ldots, X_{(k-1)}>X_{(k-2)}+1, X_{(k-1)}<N-1\right) .
$$

Also, note that the joint probability density function of $\left(X_{(1)}, \ldots, X_{(k-1)}\right)$ equals $(k-1)$ ! on the $\operatorname{set}\left\{X_{(1)}>1, X_{(2)}>X_{(1)}+1, \ldots, X_{(k-1)}>X_{(k-2)}+1, X_{(k-1)}<N-1\right\}$. Let $Y_{i}:=X_{(i)}-i$ for each $i=1, \ldots, k-1$. Then (5) equals

$$
\mathbb{P}\left(Y_{1}>0, Y_{2}>Y_{1}, \ldots, Y_{k-1}>Y_{k-2}, Y_{k-1}<N-k\right),
$$

where the joint probability density function of $\left(Y_{1}, \ldots, Y_{k-1}\right)$ has the value $(k-1)$ ! on the set $\left\{Y_{1}>0, Y_{2}>Y_{1}, \ldots, Y_{k-1}>Y_{k-2}, Y_{k-1}<N-k\right\}$. Observe that this means that $\left(Y_{1}, \ldots, Y_{k-1}\right)$ has the same distribution as the order statistics of $k-1$ uniform $[0, N]$ random variables when their largest value is smaller than $N-k$, and the probability of this happening obviously equals $(1-k / N)^{k-1}$, thus obtaining (1).

It is a matter of an easy verification to check that the above arguments generalize to the case of distinct disk sizes. In addition, suppose that the disk sizes $E_{1}, \ldots, E_{k}$ are independent and identically distributed random variables. We thus obtain

$$
\pi(k, N)=\mathbb{E}\left(\max \left\{0,1-\frac{1}{N} \sum_{i=1}^{k} E_{i}\right\}^{k-1}\right) .
$$

In both the square-root and linear scaling, asymptotics can be derived in the spirit of those that we identified for constant disk sizes. We briefly sketch how this can be done.

- First focus on the case $k=a \sqrt{N}$. Let $\bar{E}_{i}$ be the sample mean of $i$ disk sizes. Then the following inequality holds, with $\mu<\infty$ the mean disk size:

$$
\begin{aligned}
\mathbb{E}((1 & \left.\left.-\frac{a}{\sqrt{N}} \bar{E}_{a \sqrt{N}}\right)^{a \sqrt{N}-1} ; \bar{E}_{a \sqrt{N}} \in(\mu-\varepsilon, \mu+\varepsilon)\right) \\
\leq & \pi(a \sqrt{N}, N) \\
\leq & \mathbb{E}\left(\left(1-\frac{a}{\sqrt{N}} \bar{E}_{a \sqrt{N}}\right)^{a \sqrt{N}-1} ; \bar{E}_{a \sqrt{N}} \in(\mu-\varepsilon, \mu+\varepsilon)\right) \\
& +\mathbb{P}\left(\bar{E}_{a \sqrt{N}} \notin(\mu-\varepsilon, \mu+\varepsilon)\right) .
\end{aligned}
$$

It is now straightforward to see that if $\bar{E}_{N}$ obeys a law of large numbers then, as $N \rightarrow \infty$,

$$
\pi(a \sqrt{N}, N) \rightarrow \mathrm{e}^{-a^{2} \mu}
$$


- In the linear scaling, i.e. $k=a N$, we have the following lower bound:

$$
\pi(a N, N) \geq(\max \{0,1-x\})^{a N-1} \mathbb{P}\left(\bar{E}_{a N} \in(x, x+\varepsilon)\right) .
$$

Supposing that $\bar{E}_{N}$ obeys a large deviation principle with rate function $I(\cdot)$, and observing that the above lower bound applies for any $x \in(0,1)$, we obtain, after sending $\varepsilon \downarrow 0$,

$$
\liminf _{N \rightarrow \infty} \frac{1}{N} \log \pi(a N, N) \geq \sup _{x \in[0,1]}\left(a \log (1-x)-a I\left(\frac{x}{a}\right)\right) .
$$

To obtain the corresponding upper bound, realize that

$$
\begin{aligned}
\pi(a N, N) & \leq \sum_{i=1}^{m}\left(1-\frac{i-1}{m}\right)^{a N-1} \mathbb{P}\left(\frac{i-1}{a m} \leq \bar{E}_{a N}<\frac{i}{a m}\right) \\
& \leq m \max _{i=1, \ldots, m}\left(1-\frac{i-1}{m}\right)^{a N-1} \mathbb{P}\left(\frac{i-1}{a m} \leq \bar{E}_{a N}<\frac{i}{a m}\right) .
\end{aligned}
$$

Consequently,

$$
\begin{aligned}
& \limsup _{N \rightarrow \infty} \frac{1}{N} \log \pi(a N, N) \\
& \quad \leq \max _{i=1, \ldots, m}\left(a \log \left(1-\frac{i-1}{m}\right)+\limsup _{N \rightarrow \infty} \frac{1}{N} \log \mathbb{P}\left(\frac{i-1}{a m} \leq \bar{E}_{a N}<\frac{i}{a m}\right)\right) .
\end{aligned}
$$

Application of the large deviations principle yields, after sending $m \rightarrow \infty$, the counterpart of (7). We thus obtain

$$
\lim _{N \rightarrow \infty} \frac{1}{N} \log \pi(a N, N)=\sup _{x \in[0,1]}\left(a \log (1-x)-a I\left(\frac{x}{a}\right)\right) .
$$

Considering, for instance, the case that the $E_{i}$ have an exponential distribution with mean $1 / \lambda$, and $a=1$, we find that the optimizing $x$ equals

$$
x^{\star}=\frac{1}{2}+\frac{1}{\lambda}-\Lambda \in\left(0, \min \left\{\frac{1}{\lambda}, 1\right\}\right), \quad \Lambda:=\sqrt{\frac{1}{4}+\frac{1}{\lambda^{2}}},
$$

so that

$$
\lim _{N \rightarrow \infty} \frac{1}{N} \log \pi(a N, N)=\log \left(\frac{1}{2}-\frac{1}{\lambda}+\Lambda\right)+\log \left(\frac{1}{2}+\frac{1}{\lambda}-\Lambda\right)-\frac{\lambda}{2}+\lambda \Lambda+\log \lambda .
$$

Remark 3. Several further extensions can be thought of, for instance, the situation of a random number of disks. An interesting special case concerns the situation that the number of disks has a Poisson distribution. It is not hard to prove that if the mean of this Poisson distribution is $a \sqrt{N}$ then (6) still applies. If the mean is $a N$ then a standard large deviations argument yields

$$
\lim _{N \rightarrow \infty} \frac{1}{N} \log \pi(a N, N)=\sup _{b \geq 0}\left(-b \log \frac{b}{a}+b-a+\sup _{x \in[0,1]}\left(b \log (1-x)-b I\left(\frac{x}{b}\right)\right)\right) .
$$

The first terms between the brackets on the right-hand side relates to the large deviations rate function of a Poisson random variable (representing the decay rate of a Poisson random variable with mean $a N$ attaining a value close to $b N$ ); the second part is the decay rate of the probability that these $b N$ disks have no overlap. 


\section{Piecewise-constant density}

In this section we consider the case that the centers of the disks are not uniformly distributed over the interval $[0, N]$, but rather have a piecewise-constant density $\varphi_{N}(\cdot)$. More concretely, let the density be $\alpha_{1} / N$ between 0 and $f_{1} N$, then $\alpha_{2} / N$ between $f_{1} N$ and $\left(f_{1}+f_{2}\right) N$, up to $\alpha_{d} / N$ between $\left(f_{1}+\cdots+f_{d-1}\right) N$ and $\left(f_{1}+\cdots+f_{d}\right) N=N$; we assume that $\sum_{i=1}^{d} f_{i} \alpha_{i}=1$ (and, obviously, $\sum_{i=1}^{d} f_{i}=1$ ).

Notational remark. From now on, we will add a subscript ' $u$ ' to denote that the corresponding probability assumes the disks' midpoints are uniformly distributed on the interval of interest. In other words, $\pi(k, N)$ refers to the nonoverlap in the nonuniform model we consider in this section, while $\pi_{\mathrm{u}}^{\prime}(k, N)$ and $\pi_{\mathrm{u}}^{\prime \prime}(k, N)$ are given through (2).

For ease we often focus on the case $d=2$, but it is easily seen that all arguments given in this section essentially carry over to $d \in\{3,4, \ldots\}$. For the case $d=2$, the number of midpoints that ends up in $\left[0, f_{1} N\right]$ has a binomial distribution with parameters $k$ and $\alpha_{1} f_{1}$. Relying on relation (3), we have the following lower bound on the nonoverlap probability:

$$
\pi(k, N) \geq \sum_{\ell=0}^{k}\left(\begin{array}{l}
k \\
l
\end{array}\right)\left(\alpha_{1} f_{1}\right)^{\ell}\left(\alpha_{2} f_{2}\right)^{k-\ell} \pi_{\mathrm{u}}^{\prime}\left(\ell, f_{1} N_{1}\right) \pi_{\mathrm{u}}^{\prime}\left(k-\ell, f_{2} N\right)
$$

we use the probabilities $\pi_{\mathrm{u}}^{\prime}\left(\ell, f_{1} N_{1}\right)$ and $\pi_{\mathrm{u}}^{\prime}\left(\ell, f_{1} N_{1}\right)$ to properly deal with midpoints that are in $\left[0, \frac{1}{2}\right]$ and $\left[N-\frac{1}{2}, N\right]$, as well as midpoints in $\left[f_{1} N-\frac{1}{2}, f_{1} N+\frac{1}{2}\right]$. A similar argument yields

$$
\pi(k, N) \leq \sum_{\ell=0}^{k}\left(\begin{array}{l}
k \\
l
\end{array}\right)\left(\alpha_{1} f_{1}\right)^{\ell}\left(\alpha_{2} f_{2}\right)^{k-\ell} \pi_{\mathrm{u}}^{\prime \prime}\left(\ell, f_{1} N_{1}\right) \pi_{\mathrm{u}}^{\prime \prime}\left(k-\ell, f_{2} N\right) .
$$

\subsection{Square-root scaling}

In this subsection we evaluate $\pi(a \sqrt{N}, N)$ for large $N$. We do so by separately establishing a lower and upper bound. First observe that, for any $\varepsilon>0$, as $N \rightarrow \infty$, by virtue of the law of large numbers,

$$
\sum_{\ell=\left(\alpha_{1} f_{1} a-\varepsilon\right) \sqrt{N}}^{\left(\alpha_{1} f_{1} a+\varepsilon\right) \sqrt{N}}\left(\begin{array}{c}
a \sqrt{N} \\
\ell
\end{array}\right)\left(\alpha_{1} f_{1}\right)^{\ell}\left(\alpha_{2} f_{2}\right)^{a \sqrt{N}-\ell} \rightarrow 1 .
$$

We obtain the following lower bound on $\pi(a \sqrt{N}, N)$, using the fact that $\pi_{\mathrm{u}}^{\prime}(k, N)$ decreases in $k$ :

$$
\begin{aligned}
& \pi_{\mathrm{u}}^{\prime}\left(\left(\alpha_{1} f_{1} a+\varepsilon\right) \sqrt{N}, f_{1} N\right) \pi_{\mathrm{u}}^{\prime}\left(a \sqrt{N}-\left(\alpha_{1} f_{1} a-\varepsilon\right) \sqrt{N}, f_{2} N\right) \\
& \times\left[\sum_{\ell=\left(\alpha_{1} f_{1} a-\varepsilon\right) \sqrt{N}}^{\left(\alpha_{1} f_{1} a+\varepsilon\right) \sqrt{N}}\left(\begin{array}{c}
a \sqrt{N} \\
\ell
\end{array}\right)\left(\alpha_{1} f_{1}\right)^{\ell}\left(\alpha_{2} f_{2}\right)^{a \sqrt{N}-\ell}\right] .
\end{aligned}
$$

We now let $N \rightarrow \infty$. The factor in the square brackets obviously goes to 1 , while Lemma 1 entails that the product of the first two factors goes to

$$
\exp \left(-\frac{\left(\alpha_{1} f_{1} a+\varepsilon\right)^{2}}{f_{1}}\right) \exp \left(-\frac{\left(\alpha_{2} f_{2} a+\varepsilon\right)^{2}}{f_{2}}\right)
$$


Similarly, the following upper bound can be established:

$$
\begin{aligned}
& \pi(k, N) \leq \pi_{\mathrm{u}}^{\prime \prime}\left(\left(\alpha_{1} f_{1} a-\varepsilon\right) \sqrt{N}, f_{1} N\right) \pi_{\mathrm{u}}^{\prime \prime}\left(a \sqrt{N}-\left(\alpha_{1} f_{1} a+\varepsilon\right) \sqrt{N}, f_{2} N\right) \\
& +\left[1-\sum_{\ell=\left(\alpha_{1} f_{1} a-\varepsilon\right) \sqrt{N}}^{\left(\alpha_{1} f_{1} a+\varepsilon\right) \sqrt{N}}\left(\begin{array}{c}
a \sqrt{N} \\
\ell
\end{array}\right)\left(\alpha_{1} f_{1}\right)^{\ell}\left(\alpha_{2} f_{2}\right)^{a \sqrt{N}-\ell}\right] .
\end{aligned}
$$

Now the term in the square brackets goes to 0 as $N \rightarrow \infty$, whereas the first term converges to

$$
\exp \left(-\frac{\left(\alpha_{1} f_{1} a-\varepsilon\right)^{2}}{f_{1}}\right) \exp \left(-\frac{\left(\alpha_{2} f_{2} a-\varepsilon\right)^{2}}{f_{2}}\right)
$$

by virtue of Lemma 1 . Now we can let $\varepsilon \downarrow 0$ in the lower and upper bounds. As indicated above, this procedure can be generalized easily to general $d$ : the notation becomes more cumbersome, but the argument is precisely the same. It leads to the following result; it is again the square of what comes out in the discrete case [5], [13].

Theorem 1. As $N \rightarrow \infty$,

$$
\pi(a \sqrt{N}, N) \rightarrow \exp \left(-a^{2} \sum_{i=1}^{d} f_{i} \alpha_{i}^{2}\right) .
$$

Remark 4. By virtue of (4), it is easily seen that the range between square-root scaling and linear scaling can be dealt with similarly. We obtain

$$
\frac{1}{N^{2 \alpha-1}} \log \pi\left(a N^{\alpha}, N\right) \rightarrow-a^{2} \sum_{i=1}^{d} f_{i} \alpha_{i}^{2}
$$

for $\alpha \in\left[\frac{1}{2}, 1\right)$. Clearly, $\pi\left(a N^{\alpha}, N\right) \rightarrow 1$ for $\alpha$ below $\frac{1}{2}$.

\subsection{Linear scaling}

In this subsection we analyze the linear scaling, given that the midpoints of the disks are sampled from the piecewise-constant density introduced above. More specifically, we characterize the exponential decay rate

$$
\zeta:=\lim _{N \rightarrow \infty} \frac{1}{N} \log \pi(a N, N) .
$$

The first result expresses $\zeta$ in terms of a $(d-1)$-dimensional variational problem. Define $\bar{F}:=1-F$, with $F:=\sum_{i=1}^{d-1} f_{i}$. The proof relies on the 'principle of the largest term' (as in [13]), together with appropriate use of the probabilities $\pi_{\mathrm{u}}^{\prime}$ and $\pi_{\mathrm{u}}^{\prime \prime}$ (as in the proof of Theorem 1); as it is relatively straightforward, we do not include it here.

Theorem 2. For $d \in\{2,3, \ldots\}$, the decay rate $\zeta$ of $\pi(a N, N)$ equals

$$
\begin{aligned}
\zeta=-\inf _{\ell_{1}, \ldots, \ell_{d-1} \in I}( & \sum_{i=1}^{d-1} \ell_{i} \log \left(\frac{\ell_{i}}{a f_{i} \alpha_{i}}\right)+\left(a-\sum_{i=1}^{d-1} \ell_{i}\right) \log \left(\frac{a-\sum_{i=1}^{d-1} \ell_{i}}{a\left(1-\sum_{i=1}^{d-1} f_{i} \alpha_{i}\right)}\right) \\
& \left.-\sum_{i=1}^{d-1} \ell_{i} \log \left(\frac{f_{i}-\ell_{i}}{f_{i}}\right)-\left(a-\sum_{i=1}^{d-1} \ell_{i}\right) \log \left(\frac{\bar{F}-a+\sum_{i=1}^{d-1} \ell_{i}}{\bar{F}}\right)\right),
\end{aligned}
$$


where

$$
I:=\left\{\ell: 0 \leq \ell_{i} \leq f_{i} \text { for } i=1, \ldots, d-1 ; a-\bar{F} \leq \sum_{i=1}^{d-1} \ell_{i} \leq a\right\} .
$$

Note that $I$ is nonempty; it contains the vector $\ell$ with $\ell_{i}:=a f_{i}$ (which is optimal if $\left.\alpha_{1}=\cdots=\alpha_{d}=1\right)$.

In fact, the first-order conditions associated with the above variational problem do not yield any explicit solution, unless $\alpha_{1}=\cdots=\alpha_{d}=1$. To remedy that last effect, we now consider the case in which $\alpha_{i}=1+\varphi_{i} \varepsilon$, in order to see how this perturbation affects the decay rate. To this end, we write $\zeta=\sum_{k=0}^{\infty} \beta_{k} \varepsilon^{k}$ and $\ell_{i}^{\star}=\sum_{k=0}^{\infty} \kappa_{k i} \varepsilon^{k}$, and we wish to determine the coefficients $\beta_{k}$ and $\kappa_{k i}$ for $i=1, \ldots, d$ and $k=0,1, \ldots$. These coefficients can be determined explicitly (in terms of the model parameters $\boldsymbol{f}, \boldsymbol{\varphi}$, and $a$ ) in the same way as was done in [13] for the discrete birthday problem. It turns out that $\ell_{i}^{\star}=a f_{i}+a(1-a) f_{i} \varphi_{i} \varepsilon+O\left(\varepsilon^{2}\right)$, and after a substantial amount of standard analysis, we eventually find the following result. Recall that $\zeta_{0}=a \log (1-a)$.

Theorem 3. Suppose that $\alpha_{i}=1+\varphi_{i} \varepsilon$ for $i=1, \ldots, d$. Then, as $\varepsilon \rightarrow 0$,

$$
\zeta=\zeta_{0}-\left(\sum_{i=1}^{d} f_{i}\left(\varphi_{i} \varepsilon\right)^{2}\right) a^{2}+O\left(\varepsilon^{3}\right)
$$

\section{General density}

Let $\varphi(\cdot)$ be a general density on $[0,1)$; we first assume that $\varphi(\cdot)$ is continuous (also implying that $\varphi(0)=\varphi(1)$ ), and later we indicate to what extent this requirement can be relaxed. In this section the midpoints of the disks are independent samples from a distribution with density $\varphi_{N}(x):=N^{-1} \varphi(x / N)$ on $x \in[0, N)$. Again, we separately deal with the square-root scaling and the linear scaling.

\subsection{Square-root scaling}

Our main result is the following. It can be seen as a generalization of Theorem 1.

Theorem 4. As $N \rightarrow \infty$,

$$
\pi(a \sqrt{N}, N) \rightarrow \exp \left(-a^{2} \int_{0}^{1}(\varphi(x))^{2} \mathrm{~d} x\right) .
$$

Proof. The proof intensively relies on Theorem 1. The nonoverlap probability in the model with a general (continuous) density is bounded from above and below by related nonoverlap probabilities for the model with a piecewise-constant density. We first deal with the upper bound.

Upper bound. The idea is to sample $a \sqrt{N}$ times (independently) from a distribution with density $\varphi_{N}(x)$; these represent the midpoints of the intervals of length 1 . We 'thin' these $a \sqrt{N}$ intervals as follows; due to the thinning we end up with an upper bound on the probability (use the fact that the nonoverlap probability decreases in the number of samples).

Let $J_{i}(N)$ be the interval $[(i-1) N / d, i N / d)$ and $J_{i}$ be the interval $[(i-1) / d, i / d)$, and define

$$
\varphi_{i}^{-}:=\inf _{x \in J_{i}} \varphi(x), \quad \varphi_{i, N}^{-}:=\inf _{x \in J_{i}(N)} \varphi_{N}(x)=\frac{\varphi_{i}^{-}}{N} .
$$


Suppose that one of the independently sampled midpoints falls in the interval $J_{i}(N)$; let it have the value $X$. With $U$ being an independently drawn uniform random variable on $[0,1]$, we remove this sample if $U>\varphi_{i, N}^{-} / \varphi_{N}(X)$, and keep it otherwise. It is now not hard to see that an arbitrary midpoint (out of the $a \sqrt{N}$ that have been sampled) ends up in interval $J_{i}(N)$ with probability

$$
\int_{x \in J_{i}(N)} \mathbb{P}\left(U \leq \frac{\varphi_{i, N}^{-}}{\varphi_{N}(x)}\right) \varphi_{N}(x) \mathrm{d} x=\frac{\varphi_{i}^{-}}{d},
$$

whereas the probability that the disk is removed is

$$
1-\sum_{i=1}^{d} \frac{\varphi_{i}^{-}}{d}=: \varepsilon_{d}
$$

The next observation is that, given a midpoint ends up in the $i$ th interval, i.e. $J_{i}(N)$, its position $X$ within the interval is uniformly distributed. This follows from (with $u \in J_{i}(N)$ )

$$
\begin{aligned}
\mathbb{P}\left(X \leq u \mid X \in J_{i}(N), U \leq \frac{\varphi_{i, N}^{-}}{\varphi_{N}(x)}\right) & =\frac{\int_{x \in J_{i}(N), x \leq u} \mathbb{P}\left(U \leq \varphi_{i, N}^{-} / \varphi_{N}(x)\right) \varphi_{N}(x) \mathrm{d} x}{\int_{x \in J_{i}(N)} \mathbb{P}\left(U \leq \varphi_{i, N}^{-} / \varphi_{N}(x)\right) \varphi_{N}(x) \mathrm{d} x} \\
& =\frac{u d}{N}-(i-1) .
\end{aligned}
$$

Summarizing, by performing the thinning procedure, we are with our 'thinned sample' in the situation of Section 4: a finite number of intervals on which the density is constant. It follows that, with $\varphi_{d+1}^{-}:=d-\sum_{i=1}^{d} \varphi_{i}^{-}$,

$$
\pi(a \sqrt{N}, N) \leq \sum_{\ell \in S_{N}}\left(\begin{array}{c}
a \sqrt{N} \\
\ell_{1}, \ldots, \ell_{d+1}
\end{array}\right)\left(\prod_{i=1}^{d+1}\left(\frac{\varphi_{i}^{-}}{d}\right)^{\ell_{i}}\right)\left(\prod_{i=1}^{d} \pi_{\mathrm{u}}^{\prime \prime}\left(\ell_{i}, \frac{N}{d}\right)\right),
$$

where

$$
S_{N}:=\left\{\ell \in\{0,1, \ldots\}^{d+1}: \sum_{i=1}^{d+1} \ell_{i}=a \sqrt{N}\right\} .
$$

The following step mimics the upper bound of the proof of Theorem 1, i.e. we invoke the law of large numbers. More specifically, define, for $\delta>0$ arbitrarily chosen,

$$
S_{N}(\delta):=\left\{\ell \in S_{N}: \ell_{i} \in\left(a \sqrt{N} \frac{\varphi_{i}^{-}}{d}(1-\delta), a \sqrt{N} \frac{\varphi_{i}^{-}}{d}(1+\delta)\right) \text { for } i=1, \ldots, d+1\right\} .
$$

Because of the law of large numbers, with overwhelming probability, the number of samples that end up in the various intervals will be close to $a \sqrt{N} \varphi_{i}^{-} / d$ for $i=1, \ldots, d+1$ :

$$
\sum_{\ell \in S_{N}(\delta)}\left(\begin{array}{c}
a \sqrt{N} \\
\ell_{1}, \ldots, \ell_{d+1}
\end{array}\right)\left(\prod_{i=1}^{d+1}\left(\frac{\varphi_{i}^{-}}{d}\right)^{\ell_{i}}\right) \rightarrow 1
$$

as $N \rightarrow \infty$. Obviously,

$$
\begin{aligned}
\pi(a \sqrt{N}, N) \leq & \sum_{\ell \in S_{N} \backslash S_{N}(\delta)}\left(\begin{array}{c}
a \sqrt{N} \\
\ell_{1}, \ldots, \ell_{d+1}
\end{array}\right)\left(\prod_{i=1}^{d+1}\left(\frac{\varphi_{i}^{-}}{d}\right)^{\ell_{i}}\right) \\
& +\sum_{\ell \in S_{N}(\delta)}\left(\begin{array}{c}
a \sqrt{N} \\
\ell_{1}, \ldots, \ell_{d+1}
\end{array}\right)\left(\prod_{i=1}^{d+1}\left(\frac{\varphi_{i}^{-}}{d}\right)^{\ell_{i}}\right)\left(\prod_{i=1}^{d} \pi_{\mathrm{u}}^{\prime \prime}\left(\ell_{i}, \frac{N}{d}\right)\right) .
\end{aligned}
$$


The first term on the right-hand side vanishes as $N \rightarrow \infty$ due to (8); we therefore focus on the second term. Note that, for $\ell \in S_{N}(\delta)$, we have $\ell_{i} \geq a \sqrt{N}\left(\varphi_{i}^{-} / d\right)(1-\delta)$. Recalling that the nonoverlap probabilities decrease in the number of samples, we conclude that the second term is majorized by

$$
\left(\prod_{i=1}^{d} \pi_{\mathrm{u}}^{\prime \prime}\left(a \sqrt{N} \frac{\varphi_{i}^{-}}{d}(1-\delta), \frac{N}{d}\right)\right) \sum_{\ell \in S_{N}(\delta)}\left(\begin{array}{c}
a \sqrt{N} \\
\ell_{1}, \ldots, \ell_{d+1}
\end{array}\right)\left(\prod_{i=1}^{d+1}\left(\frac{\varphi_{i}^{-}}{d}\right)^{\ell_{i}}\right) .
$$

The second factor in the above display converges to 1 as $N \rightarrow \infty$, again by virtue of (8). Due to our earlier results, the first factor converges to

$$
\prod_{i=1}^{d} \exp \left(-\frac{a^{2}}{d}\left(\varphi_{i}^{-}\right)^{2}(1-\delta)^{2}\right)=\exp \left(-\frac{a^{2}}{d} \sum_{i=1}^{d}\left(\varphi_{i}^{-}\right)^{2}(1-\delta)^{2}\right) .
$$

Now letting $\delta \downarrow 0$, we obtain

$$
\limsup _{N \rightarrow \infty} \pi(a \sqrt{N}, N) \leq \exp \left(-\frac{a^{2}}{d} \sum_{i=1}^{d}\left(\varphi_{i}^{-}\right)^{2}\right) .
$$

Letting $d \rightarrow \infty$, it is readily seen that

$$
\frac{1}{d} \sum_{i=1}^{d}\left(\varphi_{i}^{-}\right)^{2} \rightarrow \int_{0}^{1}(\varphi(x))^{2} \mathrm{~d} x
$$

This proves the upper bound.

Lower bound. Where in the upper bound we 'thinned' the number of samples (to arrive at a larger sample, each of which obeyed a piecewise-constant density), in the lower bound we have to 'expand' the number of samples. This new set of samples should again correspond to samples drawn from a piecewise-constant density, such that we are in a position to apply Theorem 1. This can be realized as follows.

Define

$$
\varphi_{i}^{+}:=\sup _{x \in J_{i}} \varphi(x), \quad \varphi_{i, N}^{+}:=\sup _{x \in J_{i}(N)} \varphi_{N}(x)=\frac{\varphi_{i}^{+}}{N}, \quad \bar{\varphi}_{i}^{+}:=\frac{\varphi_{i}^{+}}{\sum_{j=1}^{d} \varphi_{j}^{+}} .
$$

The idea is to sample the midpoints of the disks as follows: let such a midpoint be in the interval $J_{i}(N)$ with probability $\bar{\varphi}_{i}^{+}$, and let the position within the interval be uniformly distributed (that is, with density $d / N$ ). Repeat this procedure, but keep track of two counters, $K_{\text {acc }}$ and $K_{\text {rej }}$, both initialized by 0 at the beginning of the procedure. With $X$ denoting the position of an arbitrary sample (i.e. the midpoint of an arbitrary disk), increase the counter $K_{\text {acc }}$ by 1 if $U<\varphi_{N}(X) / \varphi_{i, N}^{+}$(with $U$ again being an independent random variable sampled from the uniform distribution on [0,1]), and increase $K_{\text {rej }}$ otherwise. We repeat this until $K_{\text {acc }}$ reaches the value $a \sqrt{N}$. It is now not hard to see that the positions of the samples that correspond to the $K_{\text {acc }}$ 'acceptances' are distributed with density $\varphi_{N}(\cdot)$. Define by $K$ the number of samples needed, that is, $K:=K_{\text {acc }}+K_{\text {rej }}$.

The probability $p_{d}$ that an arbitrary sample is 'accepted' is

$$
p_{d}:=\sum_{i=1}^{d} \bar{\varphi}_{i}^{+} \int_{x \in J_{i}(N)} \frac{d}{N} \frac{\varphi_{N}(x)}{\varphi_{i, N}^{+}} \mathrm{d} x=\frac{d}{\sum_{i=1}^{d} \varphi_{i}^{+}} .
$$


Observe that $K$ has a negative binomial distribution with parameters $a \sqrt{N}$ and $p_{d}$ :

$$
\mathbb{P}(K=k)=\left(\begin{array}{c}
k-1 \\
a \sqrt{N}-1
\end{array}\right) p_{d}^{a \sqrt{N}}\left(1-p_{d}\right)^{k-a \sqrt{N}} .
$$

It is now concluded, as $a \sqrt{N}$ of the $K$ samples are distributed according to $\varphi_{N}(\cdot)$, that $\pi(a \sqrt{N}, N)$ majorizes

$$
\begin{aligned}
& \sum_{k=a \sqrt{N}}^{\infty}\left(\begin{array}{c}
k-1 \\
a \sqrt{N}-1
\end{array}\right) p_{d}^{a \sqrt{N}}\left(1-p_{d}\right)^{k-a \sqrt{N}} \\
& \quad \times \sum_{\ell \in T_{N}}\left(\begin{array}{c}
k \\
\ell_{1}, \ldots, \ell_{d}
\end{array}\right)\left(\bar{\varphi}_{1}^{+}\right)^{\ell_{1}} \cdots\left(\bar{\varphi}_{d}^{+}\right)^{\ell_{d}}\left(\prod_{i=1}^{d} \pi_{\mathrm{u}}^{\prime}\left(\ell_{i}, \frac{N}{d}\right)\right),
\end{aligned}
$$

where

$$
T_{N}:=\left\{\ell \in\{0,1, \ldots\}^{d}: \sum_{i=1}^{d} \ell_{i}=k\right\}
$$

Now define

$$
T_{N}(\delta):=\left\{\ell \in T_{N}: \ell_{i} \in\left(a \sqrt{N} \frac{\varphi_{i}^{+}}{d}(1-\delta), a \sqrt{N} \frac{\varphi_{i}^{+}}{d}(1+\delta)\right) \text { for } i=1, \ldots, d\right\} .
$$

The law of large numbers yields, for any $\delta>0$,

$$
\sum_{k=a \sqrt{N}}^{\left(a / p_{d}+\delta\right) \sqrt{N}}\left(\begin{array}{c}
k-1 \\
a \sqrt{N}-1
\end{array}\right) p_{d}^{a \sqrt{N}}\left(1-p_{d}\right)^{k-a \sqrt{N}} \sum_{\ell \in T_{N}(\delta)}\left(\begin{array}{c}
k \\
\ell_{1}, \ldots, \ell_{d}
\end{array}\right)\left(\bar{\varphi}_{1}^{+}\right)^{\ell_{1}} \cdots\left(\bar{\varphi}_{d}^{+}\right)^{\ell_{d}} \rightarrow 1
$$

We obtain the lower bound

$$
\begin{aligned}
\liminf _{N \rightarrow \infty} \pi(a \sqrt{N}, N) & \geq \liminf _{N \rightarrow \infty} \prod_{i=1}^{d} \pi_{\mathrm{u}}^{\prime}\left(a \sqrt{N} \frac{\varphi_{i}^{+}}{d}(1+\delta), \frac{N}{d}\right) \\
& =\exp \left(-\frac{a^{2}}{d} \sum_{i=1}^{d}\left(\varphi_{i}^{+}\right)^{2}(1+\delta)^{2}\right) .
\end{aligned}
$$

Letting $\delta \downarrow 0$ and then $d \rightarrow \infty$ yields the result.

Remark 5. The uniform distribution essentially maximizes the nonoverlap probability, as can be seen as follows. Let $U$ be uniformly distributed on $[0,1]$. Then, due to Jensen's inequality,

$$
\int_{0}^{1}(\varphi(x))^{2} \mathrm{~d} x=\mathbb{E} \varphi^{2}(U) \geq(\mathbb{E} \varphi(U))^{2}=\left(\int_{0}^{1} \varphi(x) \mathrm{d} x\right)^{2}=1 .
$$

Then observe that the uniform density $\varphi(x)=1$ (with $x \in[0,1]$ ) actually reaches this lower bound. This result can in some sense be seen as the counterpart of the findings in [10] for the discrete birthday problem (where it was found that the discrete uniform distribution maximizes the uniqueness probability). 
Remark 6. As indicated above, it is not necessary to assume that $\varphi(\cdot)$ be continuous; in our proof of Theorem 4 , we cut the interval $[0, N]$ into $d$ equally sized pieces, but it is readily checked that this is by no means crucial for the proof. Observe that the proof still goes through, if it is assumed that there are a finite number of discontinuities.

In our proof we use that the density $\varphi(\cdot)$ be bounded. An interesting topic for future research relates to the case that $\int_{0}^{1}(\varphi(x))^{2} \mathrm{~d} x=\infty$, for instance, when $\varphi(x)=1 /(2 \sqrt{x})$; in this situation it is highly likely that the nonoverlap probability is essentially determined by the disks that fall immediately right of 0 . The main question then is: for what function $\psi(\cdot)$ does $\pi(\psi(N), N)$ have a limit in $(0,1) ?-$ it is anticipated that in this case $\psi(N) / \sqrt{N} \rightarrow 0$ as $N \rightarrow \infty$.

Remark 7. It is easily verified that the counterpart of Remark 4 applies, with $\sum_{i=1}^{d} f_{i} \alpha_{i}^{2}$ replaced by $\int_{0}^{1}(\varphi(x))^{2} \mathrm{~d} x$.

Example 1. In this example we study the effect of seasonal effects on the nonoverlap probability. Consider

$$
\varphi(x)=1+b \sin (2 m \pi x),
$$

with $b \in[0,1]$ and $m \in\{0,1, \ldots\}$. Standard computations yield

$$
\int_{0}^{1}(\varphi(x))^{2} \mathrm{~d} x=1+\frac{1}{2} b^{2} .
$$

As expected, (the approximation of) the nonoverlap probability decreases in $b$, with value $\mathrm{e}^{-3 a^{2} / 2}$ for $b=1$. Observe that the approximation is constant in $m$.

It is noted that we can construct $\varphi(\cdot)$ that lead to arbitrarily high values of $\int_{0}^{1}(\varphi(x))^{2} \mathrm{~d} x$. This is evidently the case for $\varphi(x)=m$ for $x \in[0,1 / m]$ and 0 otherwise (by sending $m$ to $\infty$ ), but we can even construct densities that are positive over the entire interval $[0,1]$. Consider, for instance, $\varphi(x)=1 /(m-1)$ for $x \in[0,1-1 / m)$ and $\varphi(x)=m-1$ for $x \in[1-1 / m, 1]$, for which $\int_{0}^{1}(\varphi(x))^{2} \mathrm{~d} x$ essentially looks like $m$.

\subsection{Linear scaling}

In this subsection we focus on the linear scaling, and determine the exponential decay rate of $\pi(a N, N)$ for the general distribution $\varphi(\cdot)$. Our main result is as follows.

Theorem 5. The decay rate $\zeta$ of $\pi(a N, N)$ equals

$$
\zeta=-\inf _{\ell \in I}\left(\int_{0}^{1} \ell(x) \log \frac{\ell(x)}{a \varphi(x)} \mathrm{d} x-\int_{0}^{1} \ell(x) \log (1-\ell(x)) \mathrm{d} x\right),
$$

where

$$
I:=\left\{\ell(\cdot) \geq 0: \int_{0}^{1} \ell(x) \mathrm{d} x=a\right\} .
$$

Proof. The proof borrows elements from the previous proofs. It combines the 'sampling ideas' of the proof of Theorem 4 with the 'principle of the largest term ideas' that were used in the proof of Theorem 2.

Upper bound. We have

$$
\pi(a N, N) \leq \sum_{\ell \in S_{N}}\left(\begin{array}{c}
a N \\
\ell_{1}, \ldots, \ell_{d+1}
\end{array}\right)\left(\prod_{i=1}^{d+1}\left(\frac{\varphi_{i}^{-}}{d}\right)^{\ell_{i}}\right)\left(\prod_{i=1}^{d} \pi_{\mathrm{u}}^{\prime \prime}\left(\ell_{i}, \frac{N}{d}\right)\right),
$$


where

$$
S_{N}:=\left\{\ell \in\{0,1, \ldots\}^{d+1}: \sum_{i=1}^{d+1} \ell_{i}=a N\right\} .
$$

Observing that the number of terms in $S_{N}$ is polynomial (and thus subexponential) in $N$, scaling $\ell$ by $N$, and relying on 'Stirling', we obtain

$$
\begin{gathered}
\zeta \leq \sup _{\ell \in S}\left(\sum_{i=1}^{d+1} \ell_{i} \log \left(\frac{a \varphi_{i}^{-}}{d \ell_{i}}\right)+\sum_{i=1}^{d} \ell_{i} \log \left(1-d \ell_{i}\right)\right), \\
S:=\left\{\ell \in \mathbb{R}_{+}^{d+1}: \sum_{i=1}^{d+1} \ell_{i}=a\right\} .
\end{gathered}
$$

The upper bound then follows by a standard limit argument $(d \rightarrow \infty)$.

Lower bound. The lower bound is very much similar to the upper bound. Observe that

$$
\begin{aligned}
\pi(a N, N) \geq & \max _{k \geq a N}\left(\begin{array}{c}
k-1 \\
a N-1
\end{array}\right) p_{d}^{k}\left(1-p_{d}\right)^{a N-k} \\
& \times \sum_{\ell \in T_{N}}\left(\begin{array}{c}
k \\
\ell_{1}, \ldots, \ell_{d}
\end{array}\right)\left(\bar{\varphi}_{1}^{+}\right)^{\ell_{1}} \cdots\left(\bar{\varphi}_{d}^{+}\right)^{\ell_{d}}\left(\prod_{i=1}^{d} \pi_{\mathrm{u}}^{\prime}\left(\ell_{i}, \frac{N}{d}\right)\right),
\end{aligned}
$$

where

$$
T_{N}:=\left\{\ell \in\{0,1, \ldots\}^{d}: \sum_{i=1}^{d} \ell_{i}=a N\right\} .
$$

Scaling $\ell$ by $N$, 'Stirling', and letting $d \rightarrow \infty$ yields the result.

So far, we have concentrated on the probability of no overlaps, but several related metrics can be analyzed as well. One could, for instance, wonder what fraction of the circle has not been covered at all, or is covered by just one disk, etc. The following result characterizes the distribution of the disks over the interval $[0, N]$. Note that here it is not required that $a$ be smaller than 1 . With $D_{i}$ denoting the $i$ th disk, define

$$
F_{j}(N):=\int_{0}^{N} 1\left\{\#\left\{i: t \in D_{i}\right\}=j\right\} \mathrm{d} t .
$$

Informally, $F_{j}(N)$ is the aggregated length of the intervals that are covered by $j$ disks. We evidently have $\sum_{j=0}^{k} F_{j}(N)=N$.

Theorem 6. As $N \rightarrow \infty$,

$$
\frac{F_{j}(N)}{N} \rightarrow \beta_{j}:=\int_{0}^{1} \frac{(a \varphi(x))^{j}}{j !} \mathrm{e}^{-a \varphi(x)} \mathrm{d} x .
$$

Proof. First observe that

$$
F_{j}(N)=\int_{0}^{N}\left(\begin{array}{c}
a N \\
j
\end{array}\right)\left(\int_{x-1 / 2}^{x+1 / 2} \varphi_{N}(y) \mathrm{d} y\right)^{j}\left(1-\int_{x-1 / 2}^{x+1 / 2} \varphi_{N}(y) \mathrm{d} y\right)^{a N-j} \mathrm{~d} x,
$$


where the integration interval with respect to $y$ should be taken modulo $N$. This can alternatively be written as

$$
\int_{0}^{1} N\left(\begin{array}{c}
a N \\
j
\end{array}\right)\left(\int_{x N-1 / 2}^{x N+1 / 2} \varphi_{N}(y) \mathrm{d} y\right)^{j}\left(1-\int_{x N-1 / 2}^{x N+1 / 2} \varphi_{N}(y) \mathrm{d} y\right)^{a N-j} \mathrm{~d} x .
$$

Observe that

$$
N \int_{x N-1 / 2}^{x N+1 / 2} \varphi_{N}(y) \mathrm{d} y \rightarrow \varphi(x)
$$

The stated follows from

$$
\frac{1}{N^{j}}\left(\begin{array}{c}
a N \\
j
\end{array}\right) \rightarrow \frac{a^{j}}{j !}
$$

in conjunction with

$$
\left(1-\int_{x N-1 / 2}^{x N+1 / 2} \varphi_{N}(y) \mathrm{d} y\right)^{a N-j} \rightarrow \mathrm{e}^{-a \varphi(x)}
$$

Observe that $\sum_{j=0}^{\infty} \beta_{j}=1$, as desired.

As an aside, we mention that $k \beta_{k} /\left(\sum_{j=1}^{\infty} j \beta_{j}\right)$ is the fraction $(N \rightarrow \infty)$ of disks that has an overlap with $k-1$ other disks.

Example 2. Consider the family of densities given by

$$
\varphi(x)= \begin{cases}\frac{1}{4} b-b x+1 & \text { for } x \in\left[0, \frac{1}{2}\right] \\ -\frac{3}{4} b+b x+1 & \text { for } x \in\left[\frac{1}{2}, 1\right]\end{cases}
$$

here $b$ ranges from 0 to 4 , where $b=0$ corresponds to the uniform density, and the density becomes more 'heterogeneous' with increasing $b$. Straightforward algebra yields

$$
\beta_{j}=\frac{2}{a b}\left(\sum_{n=0}^{j} \mathrm{e}^{-(1-b / 4) a} \frac{((1-b / 4) a)^{n}}{n !}-\sum_{n=0}^{j} \mathrm{e}^{-(1+b / 4) a} \frac{((1+b / 4) a)^{n}}{n !}\right) .
$$

In particular,

$$
\beta_{0}=\frac{2 \mathrm{e}^{-a}}{a b}\left(\mathrm{e}^{b / 4}-\mathrm{e}^{-b / 4}\right) .
$$

It is easy to see that $\beta_{0}$ increases in $b$, as could be expected on intuitive grounds: the fraction of the circle not covered by any disk increases when the density $\varphi(\cdot)$ becomes more 'peaked' as the $a N$ disks tend to be more clustered. A similar effect is observed when considering the approximation for $\pi(a \sqrt{N}, N)$ :

$$
\exp \left(-a^{2} \int_{0}^{1} \varphi^{2}(x) \mathrm{d} x\right)=\exp \left(-a^{2}\left(1+\frac{1}{48} b^{2}\right)\right)
$$

in other words, this approximation of the nonoverlap probability decreases from $\mathrm{e}^{-a^{2}}$ for $b=0$ to $\mathrm{e}^{-4 a^{2} / 3}$ for $b=4$. 


\section{Concluding remarks}

In this paper we considered the nonoverlap probability of disks on the circle, with a special focus on the situation that the locations of the disks are independently but not uniformly sampled. In various limiting regimes we succeeded in finding the asymptotics of the probability of interest.

Many problems remain open; we mention a few here.

(a) Where this model relates to overlap in a one-dimensional setting, in various application areas (such as wireless networking) one would be interested in their multidimensional counterparts.

(b) A dynamic variant of our model could be considered, in which disks arrive according to a Poisson process (with rate $\lambda$ ), are accepted if they do not overlap with the disks present, and leave after a random time (with mean $1 / \mu$ ). In the discrete birthday problem, it is an easy exercise to see that the number of items present has in equilibrium a binomial distribution with parameters $N$ and $\varrho /(\varrho+N)$, with $\varrho:=\lambda / \mu$, but the answer in our continuous setting has not been found so far.

(c) We observed that the nonoverlap probability decays essentially exponentially under the linear scaling. This raises the question of whether it is possible to develop an asymptotically efficient simulation scheme for estimating this probability in the situation that the event of interest is rare, for instance, based on importance sampling.

\section{Acknowledgements}

The authors wish to thank Deepak Dhar and Satya Mazumdar for useful inputs on the related literature in statistical physics. They wish to thank Daryl Daley, Gennadi Samorodnitsky, and Rajesh Sundaresan for useful advice. MM wishes to thank Marcus Klaas de Vries for carefully reading the manuscript.

\section{References}

[1] Abramson, M. and Moser, W. O. J. (1970). More birthday surprises. Amer. Math. Monthly 77, 856-858.

[2] Barbour, A. D., Holst, L. And Janson, S. (1992). Poisson Approximation. Oxford University Press, New York.

[3] Bartelt, M. C. and Privman, V. (1991). Kinetics of irreversible monolayer and multilayer adsorption. Internat. J. Modern Phys. B 5, 2883-2907.

[4] Cadilhe, A., Araujo, N. A. M. and Privman, V. (2007). Random sequential adsorption: from continuum to lattice and pre-patterned substrates. J. Phys. Condensed Matter 19, 065124, 12 pp.

[5] Camarri, M. and Pitman, J. (2000). Limit distributions and random trees derived from the birthday problem with unequal probabilities. Electron. J. Prob. 5, 18 pp.

[6] Diaconis, P. and Mosteller, F. (1989). Methods for studying coincidences. J. Amer. Statist. Assoc. 84, 853861.

[7] Evans, J. W. (1993). Random and cooperative sequential adsorption. Rev. Modern Phys. 65, 1281-1329.

[8] Gail, M. H., Weiss, G. H., Mantel, N. and O’Brien, S. J. (1979). A solution to the generalized birthday problem with application to allozyme screening for cell culture contamination. J. Appl. Prob. 16, $242-251$.

[9] Gürsey, F. (1950). Classical statistical mechanics of a rectilinear assembly. Proc. Camb. Phil. Soc. 46, $182-194$.

[10] Joag-Dev, K. and Proschan, F. (1992). Birthday problem with unlike probabilities. Amer. Math. Monthly 99, $10-12$.

[11] KLотz, J. (1979). The birthday problem with unequal probabilities. Tech. Rep. 59, Department of Statistics, University of Wisconsin.

[12] Lieb, E. H. And Mattis, D. C. (eds) (1966). Mathematical Physics in One Dimension: Exactly Soluble Models of Interacting Particles. Academic Press.

[13] Mandjes, M. (2011). Generalized birthday problems in the large-deviations regime. Preprint.

[14] NunnikHoven, T. S. (1992). A birthday problem solution for nonuniform birth frequencies. Amer. Statistician 46, 270-274. 
[15] RÉNYI, A. (1958). On a one-dimensional problem concerning random space-filling. Publ. Math. Inst. Hung. Acad. Sci. 3, 109-127.

[16] Salsburg, Z. W., Zwanzig, R. W. and Kirkwood, J. G. (1953). Molecular distribution functions in a onedimensional fluid. J. Chem. Phys. 21, 1098-1107.

[17] Takahashi, H. (1942). Eine einfache Methode zur Behandlung der statistischen Mechanik eindimensionaler Substanzen. Proc. Phys. Math. Soc. Japan 24, 60. Reprinted in Mathematical Physics in One Dimension, eds E. Lieb and D. Mattis, Academic Press, New York, 1966, pp. 25-27.

[18] Tonks, L. (1936). The complete equation of state of one, two and three-dimensional gases of hard elastic spheres. Phys. Rev. 50, 955-963.

[19] Von Mises, R. (1938). Über Aufteilungs- und Besetzungswahrscheinlichkeiten. Rev. Fac. Sci. Univ. Istanbul 4, 145-163. 